\title{
RETRACTED ARTICLE: Comprehensive characterization of tumor mutation burden in clear cell renal cell carcinoma based on the three independent cohorts
}

\author{
Jing Huang ${ }^{1} \cdot$ Zhou Li $^{2} \cdot$ Lijun Fu $^{1}$ - Dahe Lin ${ }^{1}$. Chunhua Wang ${ }^{1}$ Xiumei Wang ${ }^{1}$ - Lifen Zhang ${ }^{1}$
}

Received: 10 October 2019 / Accepted: 10 January 2020 / Published online: 2 July 2020

(c) Springer-Verlag GmbH Germany, part of Springer Nature 2020

The Editor-in-Chief has retracted this article. Following publication, concerns were raised regarding the ownership of the data. The corresponding author has stated that some of the data were obtained from a third party, and upon request has been unable to provide the raw data. The Editor-in-Chief therefore considers the article to be unreliable.

Jing Huang, Lijun Fu and Xiumei Wang agree to this retraction; Zhou Li, Dahe Lin, Chunhua Wang and Lifen
Zhang, have not responded to any correspondence from the editor/publisher about this retraction.

The online version of this article contains the full text of the retracted article as Supplementary Information.

Electronic supplementary material The online version of this article (https://doi.org/10.1007/s00432-020-03299-3) contains supplementary material, which is available to authorized users.

Jing Huang

jing_huang89@sina.com

1 Fujian Provincial Key Laboratory of Ecology-Toxicological Effects and Control for Emerging Contaminants, Key Laboratory of Ecological Environment and Information Atlas (Putian University) Fujian Provincial University, Key Laboratory of Loquat Germplasm Innovation and Utilization (Putian University), Fujian Province University, College of Environmental and Biological Engineering, Putian University, Putian 351100, Fujian, China

2 School of Public Health, Southern Medical University, Guangzhou 510515, Guangdong, China 